J. Phys. IV France 138 (2006) 63-72

(C) EDP Sciences, Les Ulis

DOI: $10.1051 / \mathrm{jp} 4: 2006138008$

\title{
Source X-UV pour la spectroscopie d'absorption en régime femtoseconde
}

\author{
L. Lecherbourg ${ }^{1,2}$, S. Fourmaux ${ }^{1, *}$, J.C. Kieffer ${ }^{1}$, F. Martin ${ }^{1}$, H. Pépin ${ }^{1}$, \\ M. Chaker ${ }^{1}$, S. Magnan ${ }^{3}$ et C.Y. Coté ${ }^{3}$ \\ ${ }^{1}$ INRS-emt, Univ. du Québec, Varennes, J3X 1S2, Canada \\ ${ }^{2}$ LULI, 91192 Palaiseau, France \\ ${ }^{3}$ AXIS Photonique Inc., Varennes, J3X 1S2, Canada
}

\begin{abstract}
Résumé. Les processus dynamiques se produisant lors de transitions de phase ultra-rapide peuvent être déduits à partir de mesures de diffraction ou d'absorption de rayonnement $\mathrm{X}$. Les lasers femtosecondes ont récemment été utilisés pour étudier la dynamiques de la matière au moyen d'une pompe optique et d'une sonde $\mathrm{X}$ : du rayonnement $\mathrm{X} \mathrm{K}$ alpha produit par interaction laser plasma. Nous présentons nos plus récents résultats concernant le développement d'un sytème de spectroscopie d'absorption du rayonnement X (XAS) basée sur une source laser-plasma large bande dans la gamme 1-5 nm permettant d'atteindre une résolution temporelle femtoseconde. Le système est conçu pour sonder les dynamiques électroniques ayant lieu durant la transion de phase semiconducteur-métal du dyoxide de vanadium (VO2) lorsque celle-ci est initiée par une impulsion laser femtoseconde. Dans la présente expérience, un spectre large bande proche du seuil $\mathrm{L}$ du vanadium $(511 \mathrm{eV})$ et du seuil $\mathrm{K}$ de l'oxygène $(525 \mathrm{eV})$ du $\mathrm{VO} 2$ a été généré et mesuré avec un haut rapport signal sur bruit (100), une grande résolution spectrale $\left(\Delta \mathrm{E} / \mathrm{E}=4.2 \times 10^{-3}\right)$, et une résolution temporelle de 1,2 ps.
\end{abstract}

\section{INTRODUCTION}

Les processus dynamiques se produisant dans le domaine de la physique de la matière condensée ont récemment été étudiées sur des échelles de temps de l'ordre de la picoseconde et de la femtoseconde. Une impulsion laser femtoseconde constitue la pompe optique qui est utilisée pour initier la réaction d'intérêt dans un échantillon. L'évolution temporelle de cette réaction est ensuite mesurée, par des techniques de diffraction ou d'absorption $\mathrm{X}$, par une sonde de rayonnement $\mathrm{X}$ issue d'une source secondaire produite par interaction laser plasma. La combinaison d'un tel faisceau sonde avec la technique de diffraction $\mathrm{X}$ a été utilisée pour étudier differents mécansimes tels que : les processus ultrarapides dans des films organiques chauffés par lasers [1,2], la production cohérente de phonons dans des cristaux [3, 4], le mécanisme de fusion ultrarapide dans des semiconducteurs [5, 6, 7], et la transition de phase solide-solide femtoseconde dans le dixoyde de vanadium (VO2) [8].

La diffraction $\mathrm{X}$ utrarapide permet de sonder les électrons des couches internes qui sont fortement corrélés aux positions atomiques et donne ainsi accès a des informations structurelles avec une résolution temporelle ultime de quelques centaines de femtosecondes. Les techniques d'absorption X donnent accès quant à elle à la structure atomique locale et sont spécifiques à l'élément atomique étudié. Proche des seuils, la structure de spectroscopie d'absorption (NEXAFS: Near Edge Absorption Spectroscopy) donne accès aux dynamiques électroniques se produisant dans des niveau électroniques spécifiques car cette technique permet de sonder les états de valence innocupés à partir d'un niveau électronique interne. De plus, les techniques basées sur l'étude de la structure fine d'absorption $\mathrm{X}$ au delà du seuil (EXAFS: extended x-ray absorption fine structure) permettent d'accéder d'une manière simple à la struture atomique local dans le voisinage d'un atome d'un système complexe. Les éléments clés pour le succès de telles expériences au moyen d'une source X plasma sont les suivants : i) le contrôle de la

* Contact : fourmaux@emt.inrs.ca 
forme spectrale de la source $\mathrm{X}$, ii) l'optimisation de la gamme spectrale en fonction de l'échantillon sondé, iii) la puissance moyenne mesuré par le détecteeur afin d'optimiser le rapport signal sur bruit, iv) la résolution spectrale du système de détection, et v) la résolution temprorelle. Peu d'études ont été réalisées pour comprendre comment sont générés de tels rayonnements large bande ultrarapide $[9,10]$. La première expérience démontrant le potentiel de l'utilisation de rayonnements produit par interaction laser-plasma pour résoudre temporellement les dynamiques de la matière sur des échelles de temps picosecondes sont basées sur le NEXAFS résolue en temps [11]. Les rayonnements $\mathrm{X}$ durs émis par un plasma produit par laser ont été utilisé pour observer la dissociation $\mathrm{du}^{\mathrm{SF}_{6}}$ en phase gazeuse avec une résolution temporelle picoseconde. Dans cette expérience, le changement d'intensité d'une raie d'absorption résonnante a été mesurée en fonction du retard séparant la pompe (400 fs à $0.53 \mu \mathrm{m}$ ) de la sonde (rayonnement X de 1 ps à 2,5 keV). Plus récemment, les sources de rayonnement $\mathrm{X}$ produit par interaction laser-plasma ont été utilisées pour la spectrocopie d'absorption résolue en temps d'un plasma d'Al [12, 13], la photoexcitation d'un échantillon de silicium [14] et du XANES (X-ray absorption near edge spectroscopy) résolue en temps d'une solution d'ion $\mathrm{Fe}(\mathrm{CN})_{6}^{4}$ dans un solvant [15]. Cependant, dans ces expériences, la résolution temporelle est limitée par la durée du rayonnement X, une caméra CCD étant utilisé pour la mesure. Une méthode alternative pour améliorer la résolution temporelle est d'utiliser une caméra à balayage de fente couplée à un spectromètre. La résolution temporelle ultime obtenue avec une caméra à balayage de fente est de quelques centaines de femtoseconde en un seul tir laser et autour d'une picoseconde en mode accumulation [16, 17]. Des études de spectroscopies d'absorption de rayonnement $\mathrm{X}$ résolus en temps ont également été réalisées avec une source synchrotron avec une résolution temporelle picoseconde, par exemple sur du silicium liquide [18] ou sur des transition de phase solide-solide [19].

Nous discutons dans ce texte de la faisabilité d'un système NEXAFS femtoseconde basé sur une source de rayonnement $\mathrm{X}$ produit par interaction laser-plasma et une caméra à balayage de fente femtoseconde. Un spectre de rayonnement X large bande est généré par interaction d'un faisceau laser de haute intensité avec une cible solide. Une synchronisation parfaite est réalisée entre la pompe optique et la sonde de rayonnement $\mathrm{X}$ car les faisceaux sont issus du même système laser. Nous utilisons une caméra à balayage de fente femseconde en mode accumulation pour obtenir simultanément un bon rapport signal sur bruit et une résolution temporelle ultra-rapide, qui sont nécessaire pour réaliser la spectroscopie d'absorption X résolue en temps. Le système modèle étudié est la transition de phase ultrarapide semiconducteur-métal du dioxyde de vanadium [8] afin de valider la faisabilité de la technique d'absorption en utilisant une source X produite par laser. L'objectif est d'observer l'évolution de la structure d'absorption proche des seuils pour obtenir des informations sur les populations de bandes électroniques spécifiques. La gamme d'énergie est $500-560 \mathrm{eV}$, proche du seuil L du vanadium et du seuil $\mathrm{K}$ de l'oxygène. Des expériences de référence ont été réalisées avec le rayonnement X produit par le synchrotron ALS (Advance Ligth Source, Berkeley Lab) sur une échelle de temps picoseconde [19], et sur une échelle de temps femtoseconde [20] en utilisant la technique de découpage laser (slicing) qui permet d'isoler un flash de $300 \mathrm{fs}$ de rayonnement $\mathrm{X}$ synchrotron. Nous pensons qu'il est possible de réaliser une expérience similaire mais beaucoup plus compacte et simple en utilisant une source laser très intense et une caméra à balayage de fente comme détecteur. Il faut souligner que pour comprendre la dynamique ultrarapide du VO2 les résultats mesurés par absorption X sont complémentaires de ceux obtenus par spectroscopie optique ultrarapide [21] ou par diffraction X ultrarapide [8].

Dans la suite de ce texte, nous présentons les caractéristiques temporelles de la source de rayonnement $\mathrm{X}$ produite par laser avec notre caméra à balayage de fente PX1. Pour réaliser une expérience d'absorption de rayonnement $\mathrm{X}$ nous avons besoin d'une source de rayonnement X quasi continue où la forme spectrale et la brillance peuvent être contrôlées. De plus, le système de détection doit permettre de détecter un signal ayant $1 \%$ de fluctuation. Dans ce but, nous avons construit un spectromètre de rayonnement $\mathrm{X}$ avec une résolution spectrale $\Delta \mathrm{E} / \mathrm{E}=4 \times 10^{-3}$ et pouvant être couplé avec une caméra CCD ou notre caméra à balayage de fente PX1. Nous présentons les calibrations et tests de ce spectromètre avec le rayonnement X émis par un plasma de carbone. Nous avons démontré 
une résolution temporelle de 1,2 ps en mode accumulation avec une très grande dynamique quand le spectromètre est couplé à notre caméra à balayage de fente. Finalement, nous montrons qu'une cible de tantale donne une source de rayonnement $X$ continu intense, ce qui est très prometteur pour réaliser une expérience d'absorption résolue en temps dans la gamme 1-5 nm.

\section{CARACTÉRISTIQUES EXPÉRIMENTALES}

Les expériences ont été réalisées avec le laser femtoseconde Ti:sapphire de l'Institut National de la Recherche Scientifique (INRS). Ce système laser, très compact, est composé d'un oscillateur, d'un étireur, d'un amplificateur régénératif, et de trois étages amplification multi-passages (4, 3 et 2 passages). La compression est effectuée sous vide pour permettre d'atteindre une très forte intensité sur la cible. Le taux de répétition est de $10 \mathrm{~Hz}$ et les expériences présentées ont été réalisées avec $250 \mathrm{~mJ}$ après compression à une longueur d'onde de $800 \mathrm{~nm}$. La durée d'impulsion est de $60 \mathrm{fs}$ et la stabilité en énergie est de $3 \% \mathrm{rms}$. Le contraste (maximum de l'impulsion laser par rapport au piédestal) à la fréquence fondamentale est de $1 \times 10^{-6}$. Une correction du front de phase a été introduite sur le système pour maîtriser la qualité du faisceau et sa forme temporelle. Une optique adaptative (miroir déformable de 32 actuateurs) est utilisée à la sortie du compresseur pour contrôler les distorsions du front d'onde et la taille de la tache focale [22]. Le vide est maintenu à une valeure inférieure à $10^{-6}$ Torr. Après compression, le faisceau est doublé en fréquence avec un cristal de KDP. Une efficacité de conversion de $20 \%$ est mesurée entre la lumière à $800 \mathrm{~nm}$ et celle à $400 \mathrm{~nm}$.

Le faisceau à $400 \mathrm{~nm}(50 \mathrm{~mJ}, 60 \mathrm{fs})$ est focalisé, à l'aide d'une parabole hors axe f/3, sur une cible solide avec un angle de 68 degrés par rapport à la normale de la cible. Le diamètre de la tache focale est alors de 5 micromètres, ce qui permet d'obtenir une intensité sur cible de $10^{18} \mathrm{~W} / \mathrm{cm}^{2}$. La cible est déplacée entre deux tirs de façon à toujours disposer d'une surface vierge pour l'interaction laser-matière à chaque tir. Il est crucial d'éliminer les débris produit par la cible lors de l'interaction laser plasma car ils peuvent endommager les optiques du système ou bien se déposer sur celles-ci. Dans cet objectif, des films plastiques mobiles sont utilisés devant les optiques pour éliminer ces dommages qui dégradent l'émission de rayonnement $\mathrm{X}$.

Plusieurs cibles ont été utilisées, une cible de carbone pour la calibration spectrale, et des cibles de molybdène, d'argent, de lanthane et de tantale pour obtenir un spectre continu. Le rayonnement $\mathrm{X}$ produit par le plasma de carbone est enregistré par une diode p-i-n placée dans l'enceinte sous vide. Le rayonnement X produit à l'aide de cible d'éléments lourds est enregistré au moyen d'un PMT placé en dehors de l'enceinte sous vide et derrière une fenètre de beryllium épaisse de 50 micromètres.

Un spectromètre en réflexion dont l'élément centrale est un réseau concave à champ plan (Hitashi 001-0450, 2400 traits/mm) est utilisé pour mesurer la distribution en énergie de l'émission X produite par le plasma. Une caméra CCD ou bien la caméra à balayage peuvent facilement être utilisé de manière interchangeable à la sortie du spectromètre. Le spectromètre couvre la gamme 1-5 nm. La distance entre la source et le réseau est $237 \mathrm{~mm}$, et celle entre le réseau et le plan spectral est $235 \mathrm{~mm}$. L'angle d'incidence du rayonnement $X$ sur le réseau est 1,3 degrée. La résolution en énergie du réseau est en principe $\Delta \mathrm{E} / \mathrm{E}=4.3 \times 10^{-4}[23]$ mais comme nous allons le voir, elle est limitée par notre système de détection.

Le système de détection est placé à 45 degrée de la normale à la surface de la source X. Le spectromètre est entièrement blindé pour éviter tout bruit provenant du rayonnement directe produit par le plasma. Un filtre d'aluminium de $102,7 \mathrm{~nm}$ d'épaisseur, déposé sur une feuille de $113 \mathrm{~nm}$ de polyimide, est utilisé pour empêcher la lumière visible de pénétrer dans le spectromètre. Ce filtre est protègé par une feuille de 117,3 nm de polyimide. Des aimants sont aussi utilisés devant l'entrée du spectromètre pour dévier les électrons provenant du plasma. Le spectromètre est totalement motorisé ce qui autorise un réglage fin du positionnement du réseau quand l'enceinte expérimentale est sous vide. La caméra CCD, utilisée pour des mesures intégrées en temps, est le modèle PI-MTE 1300B à haute 
sensibilité de Princton Instruments. Elle permet d'enregistrer en une seule mesure la gamme spectrale complète de 1 à $5 \mathrm{~nm}$. Cette caméra peut facilement être remplacée, après l'alignement du réseau, par la caméra à balayage de fente PX1. Une photocathode de $\mathrm{KBr}$ a été choisi pour optimiser l'efficacité quantique autour de $500 \mathrm{eV}$. Le signal obtenu sur le phosphore à la sortie de la caméra à balayage de fente est amplifié grâce à un MCP Photeck par un facteur 2000. Le signal est ensuite enregistré par une caméra CCD Photometrics série 200. La caméra à balayage de fente est utilisée en mode accumulation synchronisée avec l'impulsion laser à l'aide d'un système de commutateur optique [17].

\section{RÉSULTATS}

La calibration du spectromètre est effectué à l'aide des raies d'émission d'un plasma de carbone et des seuils d'absorption de filtres de titane (Ti) et de polyimide placés entre la source plasma et l'entrée du spectromètre. La figure 1 montre un spectre de raies du carbone intégré dans le temps, et mesurée avec la caméra CCD sans (figure 1a) et avec (figure 1b) un filtre de $160 \mathrm{~nm}$ de titane protégé par un filtre de 118,2 nm de polyimide. Les séries hydrogènoïde et héliumoïde des raies d'émission du carbone sont identifiée sur la figure. Notre résultat est très similaire à celui obtenu par Fujikawa et al. [24] avec le même réseau et une source de rayonnement $\mathrm{X}$ produite par laser, mais présentant une durée d'impulsion piconseconde. Nous avons aussi observé les différents seuils correspondant aux éléments atomiques composant les filtres (seuil $\mathrm{K} \mathrm{du}$ carbone pour le filtre de polyimide et seuil L pour le filtre de titane). La grille rectangulaire (traits foncés) visible sur les deux figures provient du réseau et est attribuée à un défaut de fabrication.

Des coupes obtenuent à partir des graphiques de la figure 1 sont présentées dans la figure 2. La courbe du haut correspond à la mesure sans filtre de titane et celle du bas correspond au spectre obtenu avec deant le spectromètre le filtre additionnel de $160 \mathrm{~nm}$ de titane et 118,2 $\mathrm{nm}$ de polyimide. L'atténuation d'intensité observé dans la figure 2, résultant de la présence des filtres, correspond à la transmittance des deux filtres (titane et ployimide). Donc, ces données indiquent que la lumière diffusé traversant le filtre d'aluminium est limitée. Une estimation de la résolution spectrale expérimentale peut être déduite de la largeur à mi-hauteur d'une raie d'émission du carbone. Nous avons mesuré, en utilisant la largeur de la raie hydrogénoïde à $4 \mathrm{~nm}$, une résolution expérimentale $\Delta \mathrm{E} / \mathrm{E}=4.2 \times 10^{-3}$.

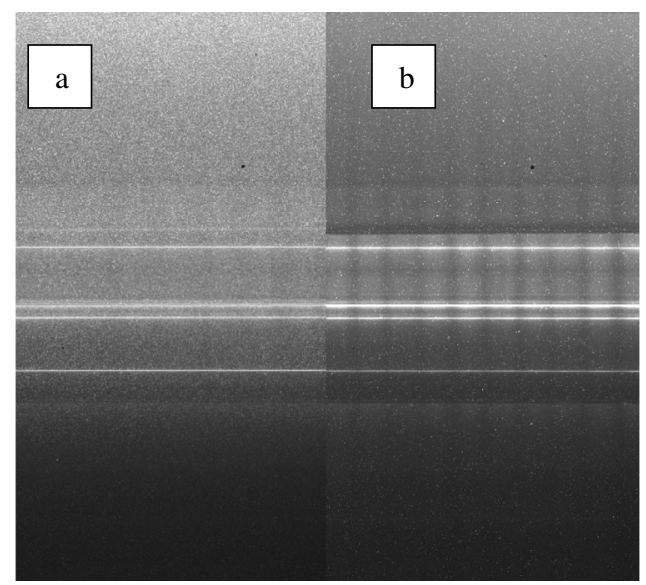

Longueur d'onde

Figure 1. Spectre intégré en temps émis par un plasma de carbone. a) spectre obtenu avec un filtre composé de $102,7 \mathrm{~nm}$ d'Al déposé sur $114 \mathrm{~nm}$ de polyimide, plus une protection d'un filtre de $117,3 \mathrm{~nm}$ de polyimide. b) spectre obtenu avec un filtre supplémentaire composé de $160 \mathrm{~nm}$ de titane et $118,2 \mathrm{~nm}$ de polyimide. 1500 tirs lasers ont été accumulé pour chaque mesure. 


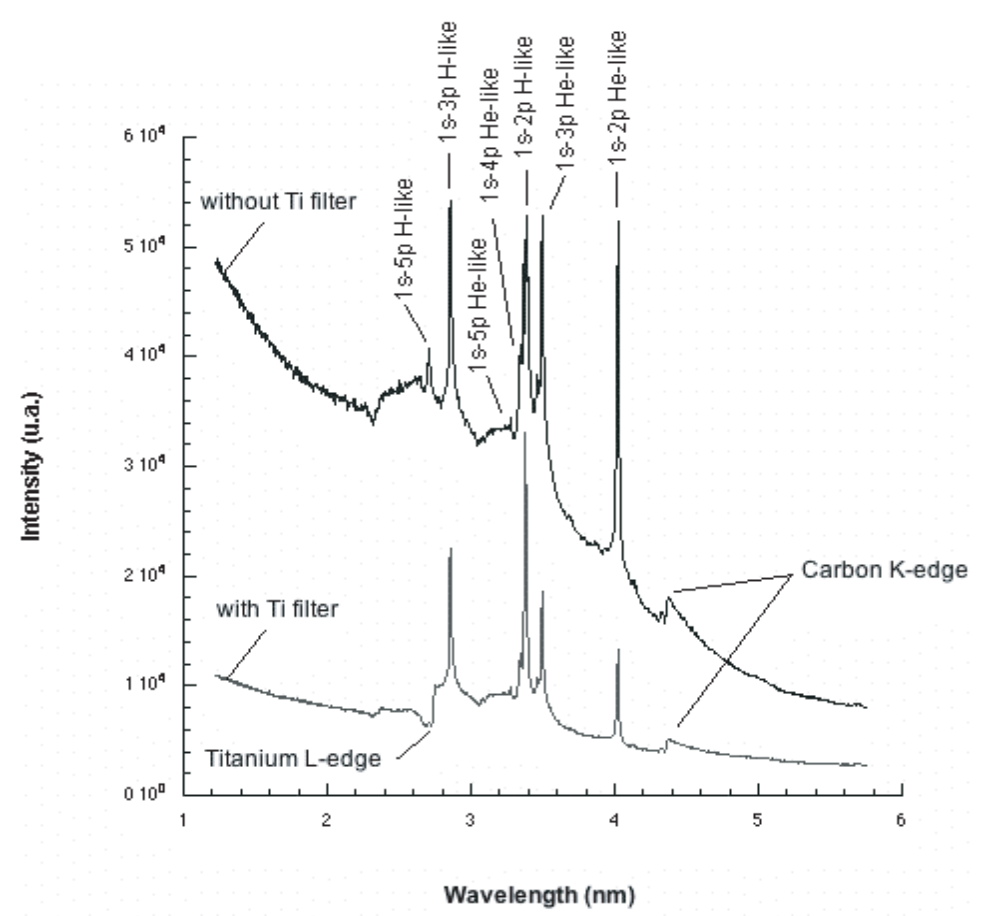

Figure 2. Spectre d'émission du carbone intégré dans le temps provenant de la figure 1. La courbe du haut (fig. 1a) est mesurée avec un filtre composé de $102,7 \mathrm{~nm}$ d'Al déposé sur $114 \mathrm{~nm}$ de polyimide, plus une protection d'un filtre de 117,3 nm de polyimide. La courbe du bas (fig. 1b ) est mesurée avec un filtre supplémentaire composé de $160 \mathrm{~nm}$ de titane et $118,2 \mathrm{~nm}$ de polyimide.

Le spectre d'émission obtenu avec la caméra à balayage de fente PX1 couplé au spectromètre est présenté dans la figure 3. L'évolution des raies hydrogémoïde et héliumoïde est observée durant quelques dizaines de picosecondes.

D'autre part, l'émission du continuum moins intense dure seulement environ 6 ps. Le balayage utilisé ici donne une dispersion temporelle de 126 fs/pixel de l'image. Le taux de monté (10\%-90\%) du spectre de rayonnement $\mathrm{X}$ est d'environ 9 pixels, ce qui nous donne une résolution temporelle autour de 1,2 ps en mode accumulation. L'évolution temporelle des raies d'émission du carbone mesurées dans notre expérience est très similaire de celle obtenue par Andiel et al. [25]. La résolution spectrale expérimentale déduite à partir de la largeur à mi-hauteur de la raie d'émission héliumoïde à $4 \mathrm{~nm}$ est inférieure à celles des spectres intégrés en temps $\left(\Delta \mathrm{E} / \mathrm{E}=9 \times 10^{-3}\right)$. Cependant, le seuil $\mathrm{K}$ du carbone du au filtre de polyimide est clairement observé sur la figure.

Dans notre gamme d'intérêt pour la réalisation d'une expérience d'absorption ultra-rapide avec un échantillon de VO2 (500-560 eV), une source de rayonnement X constituée par un plasma de tantale (Ta) est le plus prometteur en terme de brillance (figure 4). La figure 4a présente un spectre intégré en temps d'un plasma de tantale obtenu avec une caméra CCD avec (courbe du bas) et sans (courbe du haut) filtre de $160 \mathrm{~nm}$ de Ti et 118,2 $\mathrm{nm}$ de ployimide. Nous pouvons clairement observer les seuils dus aux filtres de polyimide et de titane. L'évolution temporelle de l'émission de rayonnement $\mathrm{X}$ d'un plasma de tantale mesuré avec la caméra à balayage de fente PX1 est présenté sur la figure $4 \mathrm{~b}$. Le seuil du carbone due au filtre de polyimide est clairement observé. La brillance de la source de tantale est d'un ordre de grandeur plus grande que celle de la source de carbone et donc le nombre de tir 


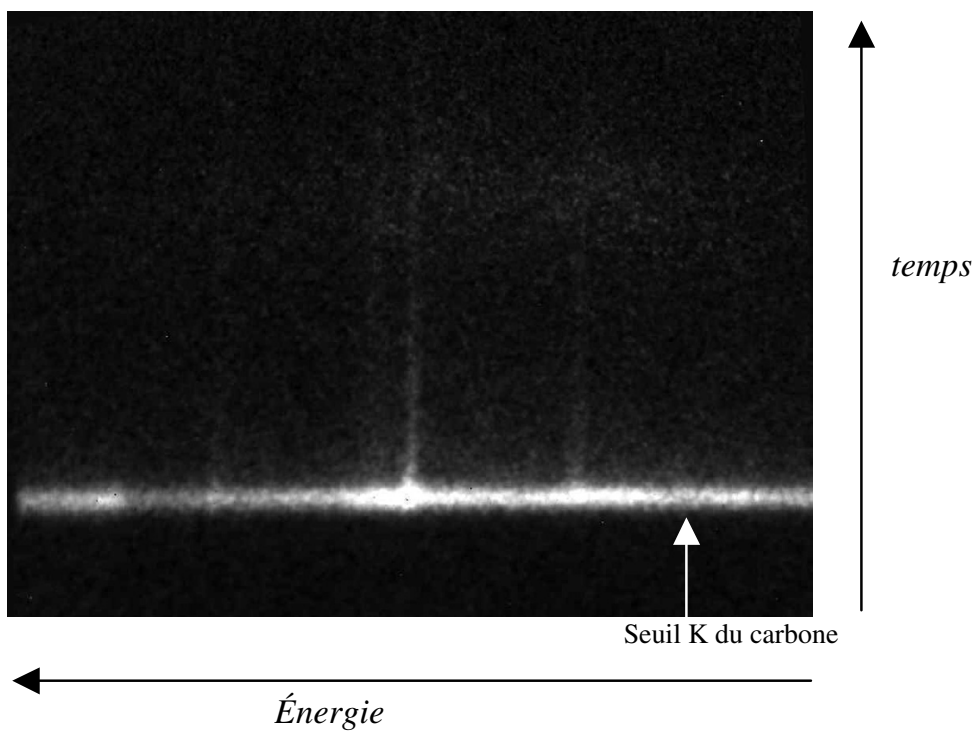

Figure 3. Spectre d'émission du carbone résolu en temps mesuré à l'aide de la caméra à balayage de fente PX1, corrigé de la courbure de la caméra. 3000 tirs lasers sont accumulés pour obtenir cette mesure. Le seuil $\mathrm{K}$ du carbone, dû au filtre de polyimide, est clairement visible.

accumulé pour obtenir cette image a été fortement diminué comparé aux résultats présenté dans la figure 3 .

Le bruit de photon peut être estimé à partir du nombre de coup mesurés il est de l'ordre de $1,2 \%$. Il peut être facilement amélioré en augmentant le nombre de tir accumulé, démontrant que ce n'est pas une limitation et ouvrant la voie pour des études NEXAFS résolues en temps dans du VO2 où un rapport signal sur bruit de $1 \%$ est nécessaire pour suivre l'évolution de la transition de phase [26].

Afin de souligner le potentiel des sources X plasma pour des études NEXAFS, nous nous sommes attardé sur le seuil $\mathrm{K}$ du carbone des filtres de polyimide. La figure 5 présente la rapport d'un spectre d'émission du tantale divisé par un spectre d'émission du tantale ayant un filtre supplémentaire de titane/polyimide autour de $300 \mathrm{eV}$. Ce résultat peut être comparé avec le spectre NEXAFS du seuil K du carbone du polyimide obtenue par Voght et al. [27]. Plusieurs structures au dessus du bruit de photon peuvent être identifiée sur notre spectre. Ce résultat préliminaire peut encore être amélioré et est très encourageant.

\section{CONCLUSION}

Pour conclure, un système NEXAFS basé sur un laser a été calibré pour sonder les dynamiques électroniques dans la transition de phase semiconducteur-métal du VO2, excitée par une impulsion laser femtoseconde. Dans la presente expérience, des spectres larges bandes proche du seuil L du vanadium et du seuil $\mathrm{K}$ de l'oxygène du VO2 $(500 \mathrm{eV})$ ont été généré et mesuré avec simultanément un haut rapport signal sur bruit (100), une haute résolution spectrale $\left(\Delta \mathrm{E} / \mathrm{E}=4 \times 10^{-3}\right)$ et une résolution temporelle de 1,2 ps. Une imnportante puissance moyenne de rayonnement $X$ peut être produite et des expériences NEXAFS femtoseconde peuvent maintenant être mise en oeuvre pour sonder un échantillon dans une géométrie pompe-sonde. 


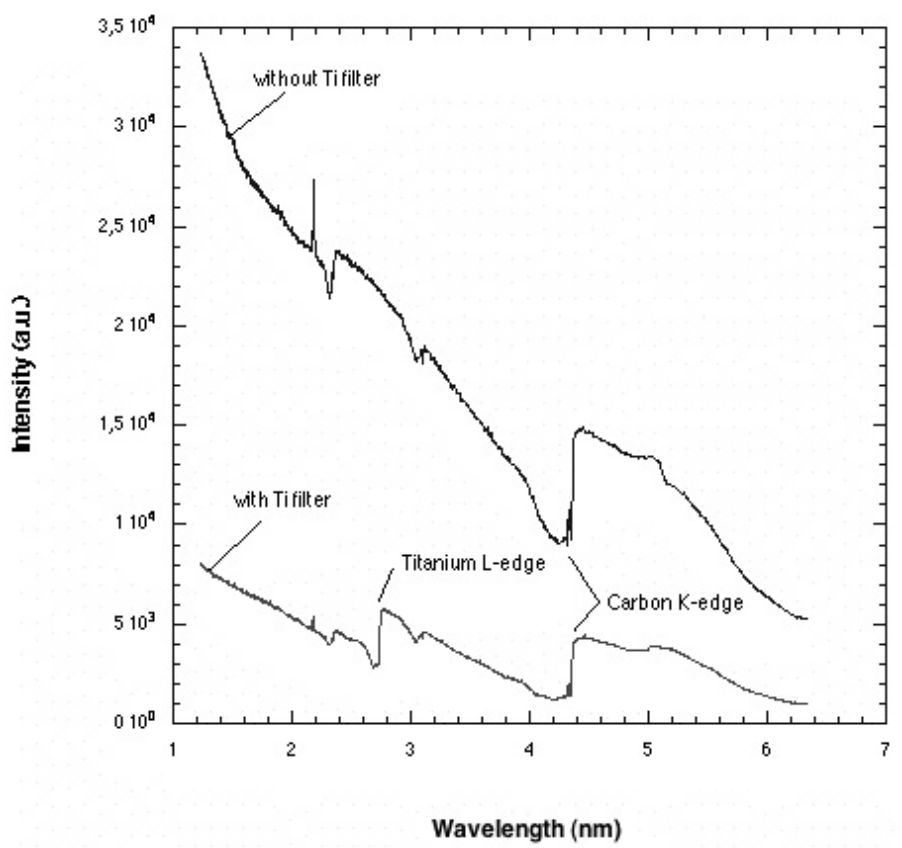

a)

b)

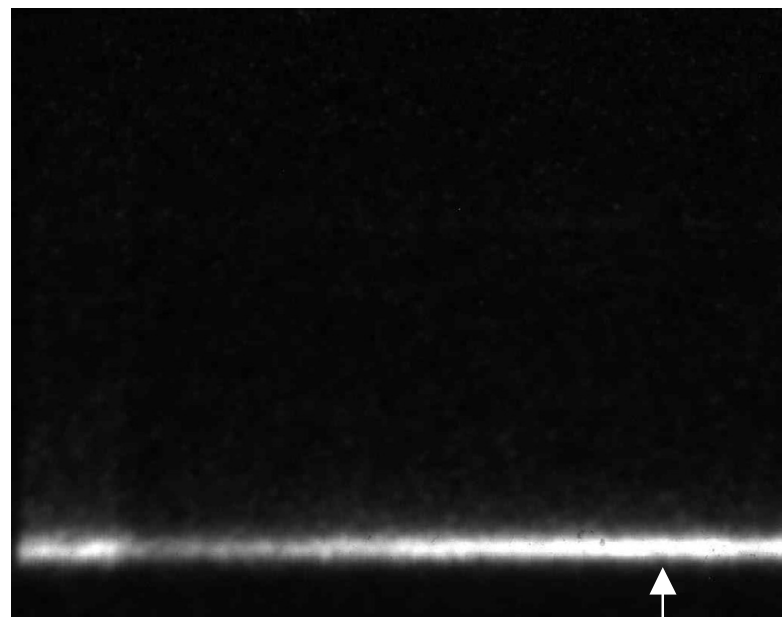

Seuil K du carbone

Énergie

temps

Figure 4. Spectre d'émission de rayonnement $X$ du tantale, corrigé de la courbure de la caméra à balayge de fente. a) Spectre intégré temporellement mesuré avec la caméra CCD. La courbe du haut est avec un filtre d'aluminium/polyimide seulement. Celle du bas est avec un filtre d'aluminium/polyimide et un filtre additionnel de titane/polyimide. 240 tirs lasers ont été accumulé pour chaque mesure. b) Spectre résolu en temps mesuré avec la caméra à balayage de fente PX1. 150 tirs lasers ont été accumulé pour obtenir cette image. Le seuil K du carbone du au filtre d'aluminium/polyimide est clairement visible. 


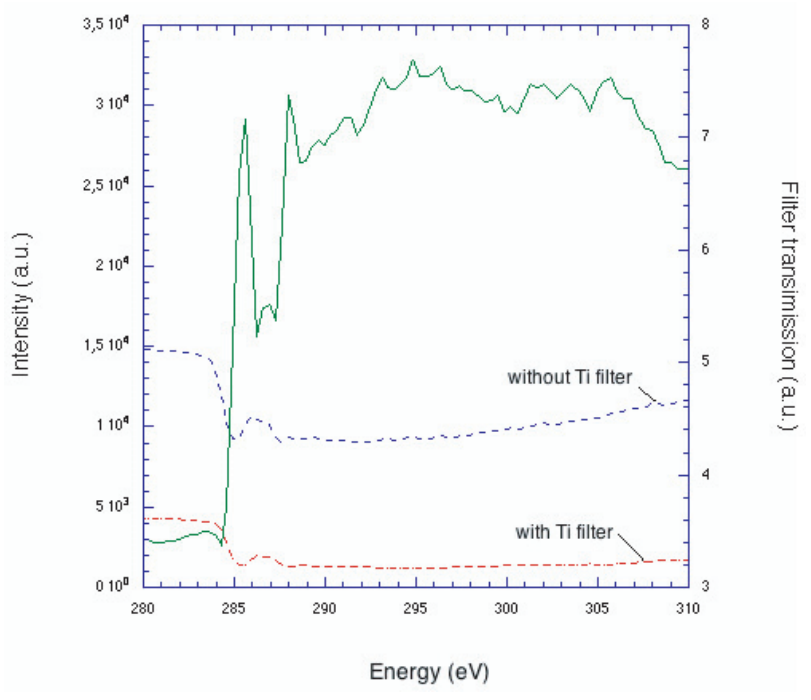

Figure 5. Rapport entre l'émission de rayonnement $\mathrm{X}$ du tantale divisé par l'émission de rayonnement $\mathrm{X}$ du tantale avec un filtre supplémentaire de titane/polyimide autour du seuil $\mathrm{K}$ du carbone à $300 \mathrm{eV}$. Les courbes en pointillées présentent l'émission du tantale provenant de la figure 4a). La courbe du haut est sans filtre supplémentaire, alors que celle du bas est avec un filtre supplémentaire de titane/polyimide.

\section{Remerciements}

Les auteurs souhaitent remercier S. Payeur, F. Poitras et C. Sirois pour leur support technique. Ce travail est financé par le NSERC, le réseau des centres d'excellences du Canada CIPI, le programme des chaires de recherche du Canada et par le Ministère de l'éducation du Québec.

\section{Références}

[1] C. Rischel, A. Rousse, I. Uschmann, P. Albouy, J. P. Geindre, P. Audebert, J. P. Gauthier, E. Forster, J. Martin, and A. Antonetti, Femtosecond time-resolved X-ray diffraction from laserheated organic films, Nature 390, 490-492 (1997)

[2] A. Rousse, C. Rischel, and J. C. Gauthier, Colloquium: Femtosecond x-ray crystallography, Reviews of Modern Physics 73, 17 (2001)

[3] C. Rose-Petruck, R. Jimenez, T. Guo, A. Cavalleri, C. W. Siders, F. Rksi, J. A. Squier, B. C. Walker, K. R. Wilson, and C. P. J. Barty, Picosecond-millingström lattice dynamics measured by ultrafast X-ray diffraction, Nature 398, 310-312 (1999)

[4] K. Sokolowski-Tinten, C. Blome, J. Blums, A. Cavalleri, C. Dietrich, A. Tarasevitch, I. Uschmann, E. Forster, M. Kammler, M. Horn-von-Hoegen, and D. von der Linde, Femtosecond $X$-ray measurement of coherent lattice vibrations near the Lindemann stability limit, Nature $\mathbf{4 2 2}$, 287-289 (2003)

[5] C. W. Siders, A. Cavalleri, K. Sokolowski-Tinten, Cs. Tóth, T. Guo, M. Kammler, M. Horn von Hoegen, K. R. Wilson, D. von der Linde, and C. P. J. Barty, Detection of Nonthermal Melting by Ultrafast X-ray Diffraction, Science 286, 1340-1342 (1999)

[6] K. Sokolowski-Tinten, C. Blome, C. Dietrich, A. Tarasevitch, M. Horn von Hoegen, D. von der Linde, A. Cavalleri, J. Squier, and M. Kammler, Femtosecond X-Ray Measurement of Ultrafast Melting and Large Acoustic Transients, Physical Review Letters 87, 225701 (2001) 
[7] A. Rousse, C. Rischel, S. Fourmaux, I. Uschmann, S. Sebban, G. Grillon, Ph. Balcou, E. Förster, J. P. Geindre, P. Audebert, J. C. Gauthier, and D. Hulin, Non-thermal melting in semiconductors measured at femtosecond resolution, Nature 410, 65-68 (2001)

[8] A. Cavalleri, Cs. Tóth, C. W. Siders, J. A. Squier, F. Ráksi, P. Forget, and J. C. Kieffer, Femtosecond Structural Dynamics in VO2 during an Ultrafast Solid-Solid Phase Transition, Physical Review Letter 87, 237401 (2001)

[9] P. Forget, F. Dorchies, J. C. Kieffer, and O. Peyrusse, Ultrafast broadband laser plasma x-ray source for femtosecond time-resolved EXAFS, Chemical Physics 299, 259 (2003)

[10] F. Shan, J. D. Carter, V. Ng, and T. Guo, Laser-driven hard-x-ray generation based on ultrafast selected energy $x$-ray absorption spectroscopy measurements of Ni compounds, Physical Review E 71, 025401 (2005)

[11] F. Ráksi, K. R. Wilson, Z. Jiang, A. Ikhlef, C. Y. Côté, and J. C. Kieffer, Ultrafast x-ray absorption probing of a chemical reaction, Journal of Chemical Physics 104, 6066 (1996)

[12] J. Workman, M. Nantel, A. Maksimchuk, and D. Umstadter, Application of a picosecond soft x-ray source to time-resolved plasma dynamics, Applied Physics Letters 70, 312 (1997)

[13] P. Audebert, P. Renaudin, S. Bastiani-Ceccotti, J.-P. Geindre, C. Chenais-Popovics, S. Tzortzakis, V. Nagels-Silvert, R. Shepherd, I. Matsushima, S. Gary, F. Girard, O. Peyrusse, and J.-C. Gauthier, Picosecond Time-Resolved X-Ray Absorption Spectroscopy of Ultrafast Aluminum Plasmas, Physical Review Letters 94, 025004 (2005)

[14] H. Nakano, Y. Goto, P. Lu, T. Nishikawa, and N. Uesugi, Time-resolved soft x-ray absorption spectroscopy of silicon using femtosecond laser plasma x rays, Applied Physics Letters 75, 2350 (1999)

[15] T. Lee, Y. Jiang, C. G. Rose-Petruck, and F. Benesch, Ultrafast tabletop laser-pump-x-ray probe measurement of solvated $\mathrm{Fe}(\mathrm{CN})_{6}^{4}$, Journal of Chemical Physics 122, 084506 (2005)

[16] P. Gallant, P. Forget, F. Dorchies, Z. Jiang, J. C. Kieffer, P. A. Jaanimagi, J. C. Rebuffie, C. Goulmy, J. F. Pelletier, and M. Sutton, Characterization of a subpicosecond $x$-ray streak camera for ultrashort laser-produced plasmas experiments, Review of Scientific Instruments 71, 3627 (2000)

[17] C. Belzile, J. C. Kieffer, C. Y. Cote, T. Oksenhendler, and D. Kaplan, Jitter-free subpicosecond streak cameras, Review of Scientific Instruments 73, 1617 (2002)

[18] S. L. Johnson, P. A. Heimann, A. M. Lindenberg, H. O. Jeschke, M. E. Garcia, Z. Chang, R. W. Lee, J. J. Rehr, and R. W. Falcone, Properties of Liquid Silicon Observed by Time-Resolved $X$-Ray Absorption Spectroscopy, Physical Review Letter 91, 157403 (2003)

[19] A. Cavalleri, H. H. W. Chong, S. Fourmaux, T. E. Glover, P. A. Heimann, J. C. Kieffer, B. S. Mun, H. A. Padmore, and R. W. Schoenlein, Picosecond soft x-ray absorption measurement of the photoinduced insulator-to-metal transition in VO2, Physical Review B 69, 153106 (2004)

[20] A. Cavalleri, M. Rini, H. H. W. Chong, S. Fourmaux, T. E. Glover, P. A. Heimann, J. C. Kieffer, and R. W. Schoenlein, Band-selective Measurement of Electronic Dynamics inVO using Femtosecond Near Edge X-ray Absorption, Physical Review Letter, To be published

[21] M. F. Becker, A. B. Buckman, R. M. Walser, T. Lépine, P. Georges, and A. Brun, Femtosecond laser excitation of the semiconductor-metal phase transition in VO2, Applied Physics Letters 65, 1507 (1994)

[22] A. V. Kudryashov, V. B. Kulakov, Y. V. Kotsuba, L. V. Novikova, V. Y. Panchenko, and V. V. Samarkin, Low-cost adaptive optical devices for multipurpose applications, SPIE Vol. 3688, 469 (1999)

[23] N. Nakano, H. Kuroda, T. Kita, and T. Harada, Development of a flat-field grazing-incidence XUV spectrometer and its application in picosecond XUV spectroscopy, Applied optics 14, 2386-2392 (1984) 
[24] C. Fujikawa, N. Yamaguchi, T. Hara, T. Kawachi, H. Oyama, K. Ando, and Y. Aoyagi, In situ calibration of a flat-field grazing incidence spectrograph with an x-ray charge-coupled device camera using a transmission grating and a laser-produced plasma for $x$-ray laser research, Review of Scientific Instruments 69, 2849 (1998)

[25] U. Andiel, K. Eidmann, and K. Witte, Time-resolved x-ray K-shell spectra from high density plasmas generated by ultrashort laser pulses, Physical Review E 63, 026407 (2001)

[26] M. Abbate, F. M. F. de Groot, J. C. Fuggle, Y. J. Ma, C. T. Chen, F. Sette, A. Fujimori, Y. Ueda, and K. Kosuge, Soft-x-ray-absorption studies of the electronic-structure changes through the VO2 phase transition, Physical Review B 43, 7263 (1991)

[27] U. Vogt, T. Wilhein, H. Stiel, H. Legall, High resolution $x$-ray absorption spectroscopy using a laser plasma radiation source, Review of Scientific Instruments 75, 4606 (2004) 